ARTICLE

\title{
Phosphorus coordinated Rh single-atom sites on nanodiamond as highly regioselective catalyst for hydroformylation of olefins
}

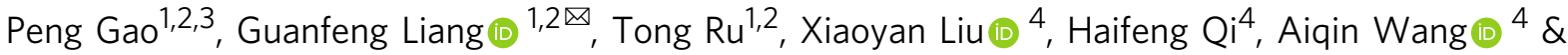 \\ Fen-Er Chen (1) 1,2,3凶
}

Single-atom Rh catalysts present superior activity relative to homogeneous catalyst in olefins hydroformylation, yet with limited success in regioselectivity control. In the present work, we develop a phosphorus coordinated $\mathrm{Rh}_{1}$ single-atom catalyst with nanodiamond as support. Benefiting from this unique structure, the catalyst exhibits excellent activity and regioselectivity in hydroformylation of arylethylenes with wide substrate generality, i.e., with high conversion (>99\%) and high regioselectivity (>90\%), which is comparable with the homogeneous counterparts. The coordination interaction between $\mathrm{Rh}_{1}$ and surface phosphorus species is clarified by ${ }^{31} \mathrm{P}$ solid-state NMR and X-ray absorption spectroscopy (XAS). Rh single atoms are firmly anchored over nanodiamond through Rh-P bonds, guaranteeing good stability in the hydroformation of styrene even after six runs. Finally, by using this catalyst, two kinds of pharmaceutical molecules, Ibuprofen and Fendiline, are synthesized efficiently with high yields, demonstrating a new prospect of single-atom catalyst in pharmaceutical synthesis.

\footnotetext{
${ }^{1}$ Engineering Center of Catalysis and Synthesis for Chiral Molecules, Department of Chemistry, Fudan University, Shanghai, China. ${ }^{2}$ Shanghai Engineering Center of Industrial Asymmetric Catalysis for Chiral Drugs, Shanghai, China. ${ }^{3}$ College of Chemistry, Sichuan University, Chengdu, Sichuan, China. ${ }^{4}$ State Key Laboratory of Catalysis, Dalian Institute of Chemical Physics, Chinese Academy of Sciences, Dalian, China. $\bigotimes_{\text {email: lianggfeng@fudan.edu.cn; rfchen@fudan.edu.cn }}$
} 


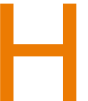
ydroformylation of olefins affords a significantly important industrial process for aldehydes synthesis ${ }^{1-5}$. Homogeneous $\mathrm{Rh}$ catalysts with various phosphorus ligands have been widely used in this process for the superior activity and selectivity ${ }^{6-12}$. Despite intensive research in homogeneous Rh-catalyzed hydroformylation, only a few examples succeeded in industry up to now ${ }^{13-16}$. Catalyst separation is an important issue, and remains challenging in most cases. Compared with homogeneous catalysts, heterogenized homogeneous $\mathrm{Rh}$ complex or supported Rh nanoparticles developed for hydroformylation have advantages in stability and catalyst recycling, but usually present much lower activities and/or selectivities ${ }^{17-22}$. Therefore, designing efficient heterogeneous $\mathrm{Rh}$ catalysts is highly desirable in this aspect.

Recently, single-atom catalysts (SACs) are emerged as a new type of heterogeneous catalysts, which have demonstrated unexpectedly high specific activity in many reactions ${ }^{23-32}$. The notable characteristic of such kind of catalyst is that active sites are atomically dispersed metal centers on supports. The remarkable reactivity of SACs was related to highly efficient use of metal atoms, and the coordination environment of metal centers with adjacent heteroatoms $(\mathrm{O}, \mathrm{N}$, or other metal atoms). The identical coordination environment of isolated metal centers often offered the selectivity comparable to homogeneous counterparts. In the pioneer work in SACs-catalyzed hydroformylation, Zhang et al. demonstrated that $\mathrm{Rh}_{1} / \mathrm{ZnO}$ nanowires SAC showed comparable activity to homogeneous Wilkinson's catalyst in the hydroformylation of styrene $e^{33}$. Although superior activity was obtained, the isolated $\mathrm{Rh}$ single atoms on $\mathrm{ZnO}$ nanowires failed to control the regioselectivity in this process, which was an important issue in hydroformylation. The ratio of branched and linear aldehydes $(b / l)$ maintained at 1:1 when $\mathrm{ZnO}$ supported $\mathrm{Rh}$ nanocluster was replaced by Rh SAC (Fig. 1a). Recently, the same group devised a $\mathrm{Rh}_{1} / \mathrm{CeO}_{2} \mathrm{SAC}$ for the hydroformylation of styrene in the presence of $\mathrm{CO}$, giving a branched/linear aldehyde $(b / l)$ ratio of $1: 3$
(Fig. 1b) ${ }^{34}$. The mechanism investigation revealed that in situ hydrogen was generated via $\mathrm{Rh}_{1} / \mathrm{CeO}_{2} \mathrm{SAC}$-catalyzed water-gas shift reaction, and exhibited a crucial role in regioselectivity. Ding and coworkers designed a series of porous organic copolymers (POPs) through polymerization of various phosphorus ligands, which served as polymer matrix to coordinate with $\mathrm{Rh}$ species $^{35-38}$. Such POPs based Rh catalysts presented high activity (TOF $>1200 \mathrm{~h}^{-1}$ ) with excellent regioselectivity to linear aldehydes $(l / b>24: 1)$ in the hydroformylation of propene. The authors claimed that partial $\mathrm{Rh}$ species were transformed into $\mathrm{Rh}$ single-atoms during reaction. Very recently, inorganic phosphorus modified $\mathrm{Rh}$ nanoparticles on $\mathrm{SiO}_{2}$ was employed in styrene hydroformylation, affording a $b / l$ molar ratio of 55:45 with an excellent turnover frequency $\left(1496 \mathrm{~h}^{-1}\right)^{39}$. Therefore, combining the advantage of regioselective homogeneous Rh-P complex and efficient heterogeneous catalysts, $\mathrm{P}$-modified $\mathrm{Rh}$ SACs might hold great potential in industrial applications. However, the successful preparation of P-coordinated metal single-atom catalyst was rarely reported. Until recently, Li and coworkers reported a graphitic P-coordinated Fe single-atom catalyst, which displayed excellent catalytic performance in hydrogenation and reductive amination reactions for the production of various amines as well as drug targets ${ }^{40}$.

Herein we report the preparation of nanodiamond-supported phosphorus coordinated $\mathrm{Rh}_{1}$ species by metal-ligand coordination, abbreviated as $\mathrm{Rh}_{1} / \mathrm{PNP}-\mathrm{ND}$, for aqueous biphasic hydroformylation reactions. In the hydroformylation of styrene, $\mathrm{Rh}_{1} /$ PNP-ND afforded the highest activity compared to other heterogenized homogenous Rh catalysts and conventional supported $\mathrm{Rh}$ catalysts. $\mathrm{Rh}_{1} / \mathrm{PNP}-\mathrm{ND}$ exhibited high regioselectivity towards branched aldehyde with a $b / l$ molar ratio up to $13.3: 1$, which outperformed all the supported Rh catalyst in the literature, and was comparable to the homogeneous counterparts (Fig. 1c). Moreover, the original activity and the regioselectivity for the branched $a$-arylpropionaldehydes still maintained after six runs,

(a) Non-regioselective $\mathrm{Rh}_{1} / \mathrm{ZnO}$ nanowires SAC

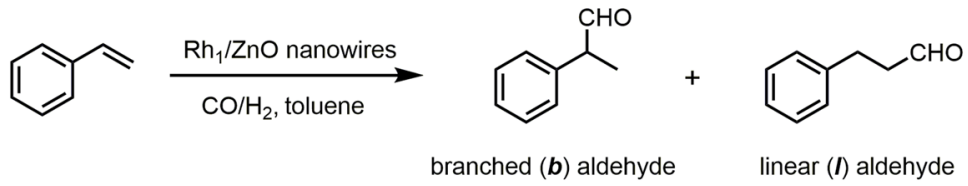

$b / l=1: 1$

(b) Linear-regioselective $\mathrm{Rh}_{1} / \mathrm{CeO}_{2} \mathrm{SAC}$

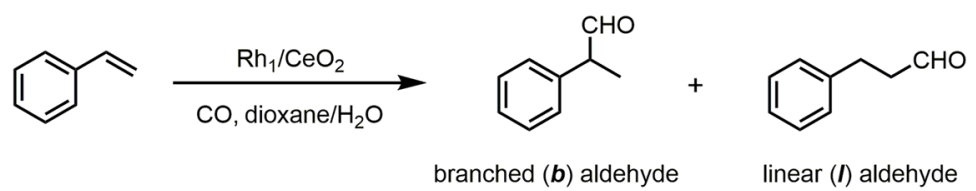

$b / l=1: 3$

(c) This work: branched-regioselective $\mathrm{Rh}_{1} / \mathrm{PNP}-\mathrm{ND}$



Vinyl arylenes

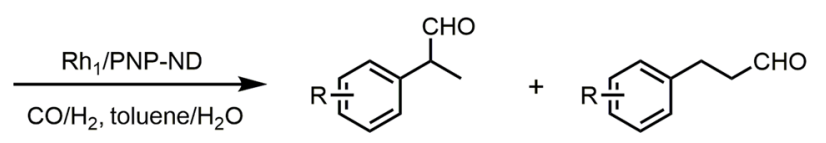

branched $(\boldsymbol{b})$ aldehydes linear $(I)$ aldehydes

b/l up to $13.3: 1$

Fig. 1 Rh single atom catalysts for regioselective hydroformylation of vinyl arylenes. a $R h_{1} / Z n O$ nanowires $S A C$. b $R h_{1} / C e O_{2} S A C$. c the present $R h_{1} /$ PNP-ND catalyst. 


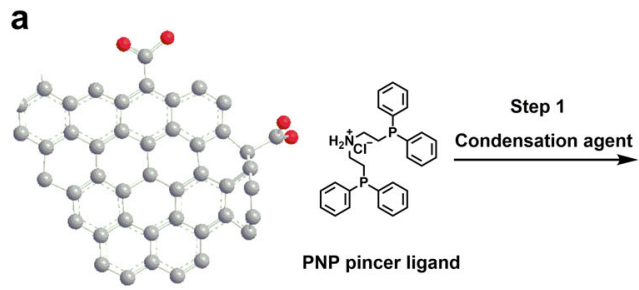

Graphene surface of ND

b

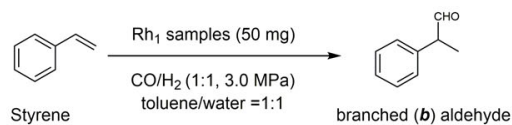

Strong stirring After stirring

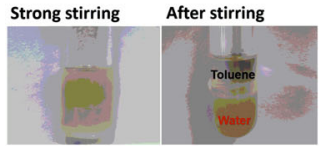

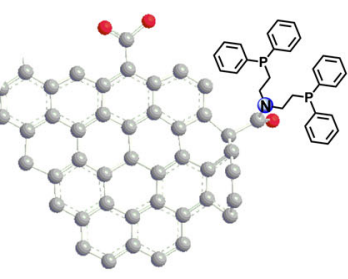

PNP-ND

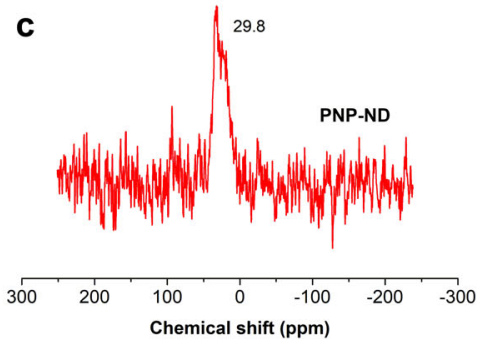

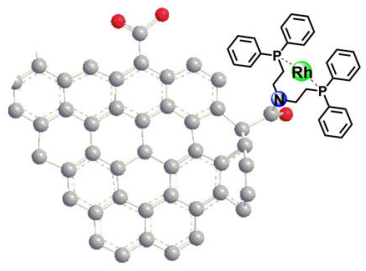

$\mathrm{Rh}_{1} / \mathrm{PNP}-\mathrm{ND}$

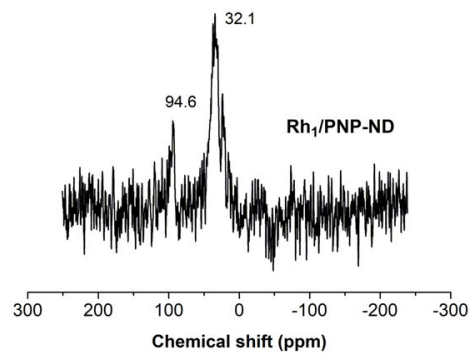

Fig. 2 The synthesis of Rh1/PNP-ND. a Schematic illustration for synthesizing Rh $/$ PNP-ND. b Catalytic hydroformylation of styrene over the synthesized Rh samples. c Solid-state ${ }^{31}$ P NMR data.

suggesting highly stable coordination structure of Rh-P. Besides, various vinyl arenes were converted to the corresponding $\alpha$ arylpropionaldehydes with remarkable catalytic activity, chemoselectivity, and regioselectivity under mild conditions, indicating the generality of the catalyst. Given the generality and regioselectivity of $\mathrm{Rh}_{1} / \mathrm{PNP}-\mathrm{ND}$, two pharmaceutical molecules Ibuprofen and Fendiline, were synthesized in a step- and atomeconomical manner with high yields.

\section{Results}

Synthesis and characterization of $\mathrm{Rh}_{\mathbf{1}} / \mathrm{PNP}-\mathrm{ND}$. Pristine and functionalized nanodiamond have been employed as catalyst or support in various catalytic transformations. Structurally, nanodiamond comprised of diamond core $\left(s p^{3}\right.$ carbon) and graphene surface ( $s p^{2}$ carbon) after annealing at high temperature. It is well known that dispersible ND possessed abundant carboxyl and hydroxyl groups on the graphene surface (further confirmed by XPS analysis in Supplementary Fig. 1b), which facilitate the surface modification with diverse organic modifiers. The synthesis procedure for $\mathrm{Rh}_{1} / \mathrm{PNP}-\mathrm{ND}$ by taking advantage of substantial carboxyl groups was illustrated in Fig. 2a (detailed information was shown in Supplementary Notes). To begin with our work, a surface modification method was used to graft PNP pincer ligand bis[2-diphenylphosphinoethyl]amine covalently on ND. In step 1, the surface carboxyl groups selectively reacted with the amino groups of PNP ligands with the catalysis of condensation agents, giving the PNPND samples. Subsequently, PNP-ND was impregnated in organic solution of $\mathrm{Rh}$ precursor (step 2). Rh species were anchored on PNP-ND via the coordination interaction with surface PNP ligand, and then reduced under hydrogen at $120^{\circ} \mathrm{C}$ for $2 \mathrm{~h}$. The catalytic performance of the Rh samples was screened in styrene hydroformylation (Fig. 2b). As shown in Supplementary Tables 1 and 2, the catalytic performances of the synthesized Rh samples were sensitive to the condensation agents, solvents, temperatures and Rh precursors in synthesis process. When NMM (N-methyl morpholine) and CDMT (2-Chloro-4,6-dimethoxy-1,3,5-triazine) was used as condensation agent in step 1 , the implantation of $[\mathrm{Rh}(\mathrm{COD}) \mathrm{Cl}]_{2}$ in THF in step 2 gave the most efficient sample (denoted as $\mathrm{Rh}_{1} /$ PNP-ND except otherwise defined), which showed the highest activity ( $>99 \%$ conversion) with $81 \%$ regioselectivity towards branched $a$-phenylpropionaldehyde $(b / l=4 \cdot 2: 1) .{ }^{31} \mathrm{P}$ solid-state NMR spectroscopy in Fig. 2c provided the direct evidence of successfully anchoring Rh species on PNP-ND. The ${ }^{31} \mathrm{P}$ NMR spectra of PNPND presented one peak at $32.1 \mathrm{ppm}$, corresponding to the PNP ligands immobilized on ND surface. By contrast, $\mathrm{Rh}_{1} / \mathrm{PNP}-\mathrm{ND}$ exhibited a major peak for immobilized PNP ligands at $32.1 \mathrm{ppm}$, and a new peak at $94.6 \mathrm{ppm}$. The ${ }^{31} \mathrm{P}$ chemical shift is a sensitive indicator of the coordination state of PNP pincer ligands ${ }^{41-43}$. The downfield shift of phosphorus resonance was attributed to the coordination of PNP pincer ligands with $\mathrm{Rh}$ though the $\mathrm{P}$ donor. This evidences PNP ligands served as hosts for Rh anchoring. X-ray photoelectron spectroscopic (XPS) measurements demonstrated the presence of $\mathrm{Rh}, \mathrm{P}$ and $\mathrm{N}$ elements on $\mathrm{Rh}_{1} / \mathrm{PNP}-\mathrm{ND}$ (Supplementary Fig. 1). The spectra of P $2 p$ displayed two peaks at 133.6 and 132.7 $\mathrm{eV}$ after deconvolution (Supplementary Fig. 1c). The former peak centered at high binding energy was attributed to $\mathrm{P}$ species coordinated with Rh, while the latter one was assigned to uncoordinated PNP. The interpretation of the C1s peak (Supplementary Fig. 1b) revealed three peaks. The peaks at 284.6 and $285.9 \mathrm{eV}$ were assigned to $s p^{2}$ and $s p^{3}$ carbon atoms respectively. The peak at $287.0 \mathrm{eV}$ represents $\mathrm{C}=\mathrm{O}$ like carbon atoms (mainly carboxyl groups). The molar ratio of $s p^{2} / s p^{3} / \mathrm{C}=\mathrm{O}$ like carbon species on the surface was 56:19:25, which revealed an enriched graphene surface with large amounts of carboxyl groups. The binding energy of Rh $3 d_{5 / 2}$ showed two peaks at 309.6 and $308.6 \mathrm{eV}$, respectively (Supplementary Fig. 1d), which is much higher than that of metallic $(306.9 \mathrm{eV})$ in literature ${ }^{33}$, indicating positively charged $\mathrm{Rh}$ state. According to the result of inductively coupled plasma atomic emission spectrometry (ICP-AES), the loading of metal Rh species on PNP-ND is $0.5 \mathrm{wt} \%$.

$\mathrm{Rh}_{1} / \mathrm{PNP}-\mathrm{ND}$ was further characterized with atomic-resolution characterization techniques. High resolution transmission electron microscopy (HRTEM) revealed that the diameter of ND particles is about 5-10 nm, and no Rh cluster was observed (Fig. 3a). In order to determine the dispersion states of $\mathrm{Rh}$ atoms, $\mathrm{Rh}_{1} / \mathrm{PNP}-\mathrm{ND}$ was investigated by the aberration-corrected high-angle annular dark-field scanning transmission electron microscopy (AC-HAADF-STEM). As shown in Fig. 3b-f, isolated bright spots were exclusively probed on $\mathrm{Rh}_{1} / \mathrm{PNP}-\mathrm{ND}$, revealing the atomically dispersed $\mathrm{Rh}$ atoms.

The X-ray adsorption fine structure (XAFS) was employed to determine the electronic states and the local coordination environment of $\mathrm{Rh}$ single atoms on $\mathrm{Rh}_{1} / \mathrm{PNP}-\mathrm{ND}$. The $\mathrm{X}$-ray absorption near-edge structure (XANES) of $\mathrm{Rh}_{1} / \mathrm{PNP}-\mathrm{ND}$ clearly showed that $\mathrm{Rh}$ species were positively charged $\left(\mathrm{Rh}^{\delta+}, 1<\delta<3\right)$, 


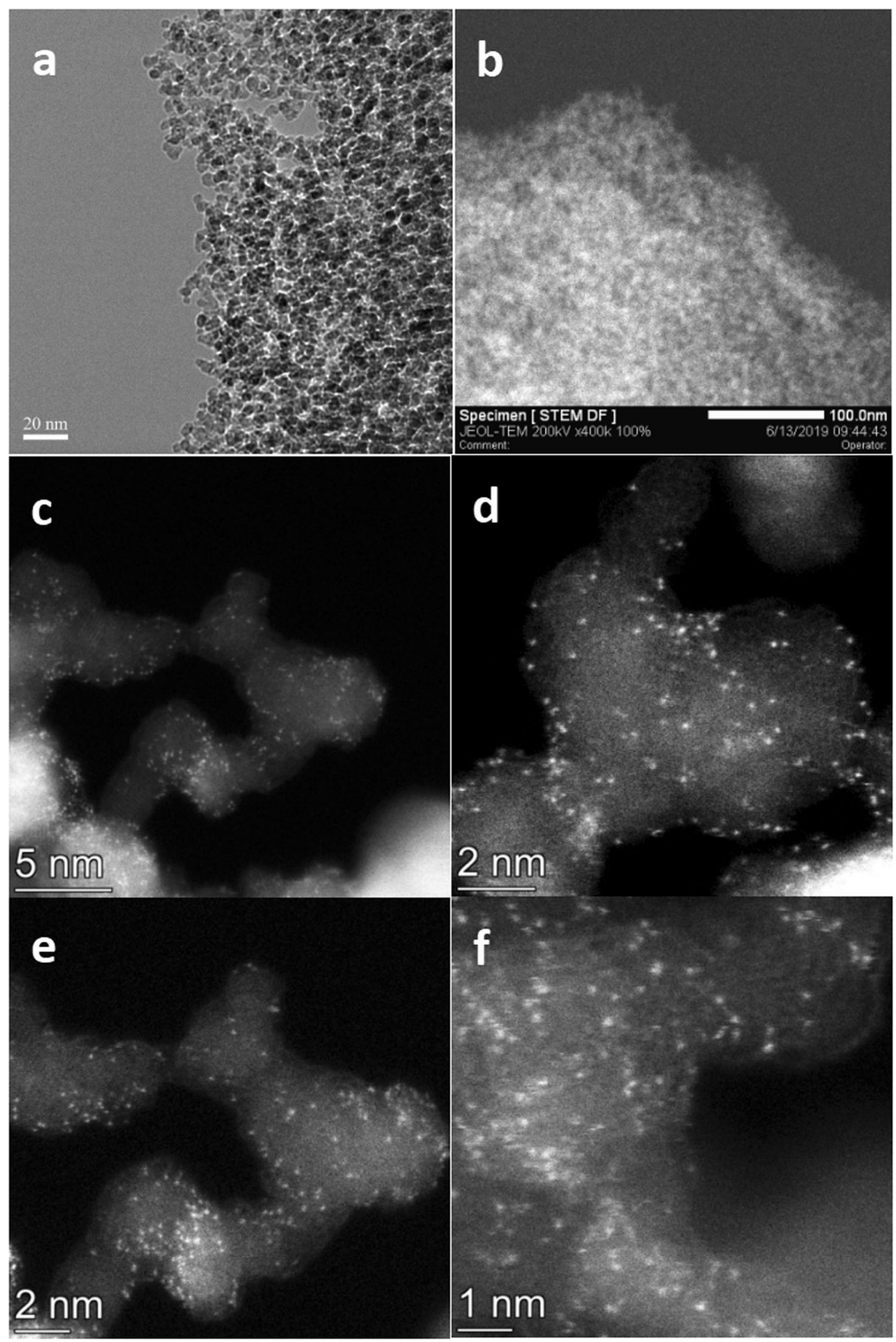

Fig. 3 Structure and Morphology characterization of Rh1/PNP-ND. a TEM image. b HAADF-STEM image. c-f AC-HAADF-STEM images.

in comparison with those of $\left[\mathrm{Rh}(\mathrm{COD}) \mathrm{Cl}_{2}\right.$ and $\mathrm{Rh}_{2} \mathrm{O}_{3}$ (Fig. 4a). It is in good agreement with the XPS spectra of $\mathrm{Rh} 3 d_{5 / 2}$ on $\mathrm{Rh}_{1} /$ PNP-ND. Furthermore, we probed the local coordination environment of isolated $\mathrm{Rh}$ atoms by using Fourier-transformed $\mathrm{k} 2$-weighted extended X-ray absorption fine structure (EXAFS) in $\mathrm{R}$ space. The sample displayed a major scattering peak at $1.5 \AA$, which was ascribed to the first coordination shell of $\mathrm{Rh}-\mathrm{C}$ or $\mathrm{Rh}-\mathrm{O}$ (Fig. 4b). In a related work recently reported by $\mathrm{Ma}$ and coworkers, similar results were obtained from the EXAFS spectra of a $\mathrm{Cu}$ single-atom catalyst on $\mathrm{ND}^{25}$. The authors decisively assigned the peak at $1.5 \AA$ to the first coordination shell of $\mathrm{Cu}-\mathrm{C}$.
In the present work the contribution of $\mathrm{Rh}-\mathrm{O}$ scattering was not excluded, considering the positively charged $\mathrm{Rh}$ species observed from XPS spectra. The unresolved peak appeared at about $1.9 \AA$ in Fig. $4 \mathrm{~b}$, suggesting the first coordination shell of Rh-P. This finding evidences that $\mathrm{Rh}$ single atoms anchored on PNP-ND were coordinated with $\mathrm{P}$ species, which has been confirmed from the results of ${ }^{31} \mathrm{P}$ solid-state NMR and XPS. The best-fitted EXAFS results of $\mathrm{Rh}_{1} / \mathrm{PNP}-\mathrm{ND}$ and the corresponding standard samples in the first shell is shown in Fig. 4c and Supplementary Table 3. $\mathrm{Rh}_{1} / \mathrm{PNP}-\mathrm{ND}$ showed a $\mathrm{Rh}-\mathrm{C} / \mathrm{O}$ coordination at $2.08 \mathrm{~A}$ with $\mathrm{CN}$ of 5.0. The $\mathrm{CN}$ of $\mathrm{Rh}$ atom with $\mathrm{P}$ atoms was 1.6, with a 



Fig. 4 The Rh XAS data of Rh1/PNP-ND and standard Rh materials. a The normalized XANES spectra at the Rh K-edge of the Rh $/ \mathrm{PNP}-\mathrm{ND}$, $[\mathrm{Rh}(\mathrm{COD})$ $\mathrm{Cl}_{2}, \mathrm{Rh}_{2} \mathrm{O}_{3}$, and Rh foil. b The Fourier transform of $\mathrm{k}^{2}$-weighted EXAFS spectra at the K-edge of the Rh1/PNP-ND, $\left[\mathrm{Rh}(\mathrm{COD}) \mathrm{Cl}_{2}, \mathrm{Rh}{ }_{2} \mathrm{O}_{3}\right.$, and $\mathrm{Rh}$ foil. $\mathbf{c}$ The experimental Rh EXAFS spectra (black line) and the fitting curve of $\mathrm{Rh}_{1} / \mathrm{PNP}-\mathrm{ND}$ (red line).

mean bond length of $2.38 \AA$, which matched approximately with the bidentate chelation of PNP pincer ligands on ND. Based on the above results, the isolated $\mathrm{Rh}$ atoms were stabilized by surrounding $\mathrm{C} / \mathrm{O}$ atoms and $\mathrm{P}$ atoms. It is well known that the chelation of phosphine, especially bidentate phosphine, was much stronger than $\mathrm{O}$ or $\mathrm{C}$ in organometallic complex, herein we proposed that bidentate phosphine of PNP-ND played a predominant role in the coordination chemistry of $\mathrm{Rh}$ single atoms.

Hydroformylation of styrene. In order to understand the superior catalytic performance of $\mathrm{Rh}_{1} / \mathrm{PNP}-\mathrm{ND}$, the pristine $\mathrm{Rh}$ PNP complex and other heterogenized Rh catalysts were prepared and screened in the hydroformylation of styrene in a toluene/ water biphasic system with syngas $\left(\mathrm{CO} / \mathrm{H}_{2}=1\right)$. As shown in Table 1, Under the standard reaction conditions (toluene $/ \mathrm{H}_{2} \mathrm{O}=$ $1: 1,3.0 \mathrm{MPa}$ syngas, $50^{\circ} \mathrm{C}$, and $10 \mathrm{~h}$ ), their catalytic performances were evaluated in terms of activity (TOF), chemoselectivity to aldehydes, and regioselectivity to branched aldehyde. The chemoselectivities for aldehydes over all the tested catalysts were $>90 \%$, especially for $\mathrm{Rh}_{1} / \mathrm{PNP}-\mathrm{ND}$, over which $>99 \%$ selectivity towards aldehydes was obtained. $\mathrm{Rh}_{1} / \mathrm{PNP}-\mathrm{ND}$ exhibited $77 \%$ conversion $\left(\mathrm{TOF}=95 \mathrm{~h}^{-1}\right.$ ) with the $b / l$ ratio up to $12.4: 1$, which outperformed all the other heterogenized $\mathrm{Rh}$ catalysts. Besides $\mathrm{Rh}_{1} / \mathrm{PNP}-\mathrm{ND}, \mathrm{Rh}-\mathrm{PNP} / \mathrm{ND}$ was synthesized though physically mixing Rh-PNP complex with unmodified ND by electrostatic adsorption. In comparison, Rh-PNP/ND gave $44 \%$ conversion $\left(\mathrm{TOF}=96 \mathrm{~h}^{-1}\right)$ with a $b / l$ ratio of $4.7: 1$. Homogeneous Rh-PNP complex gave $85 \%$ conversion $\left(\mathrm{TOF}=105 \mathrm{~h}^{-1}\right.$ ) with a $b / l$ ratio of 4.9:1. The regioselectivity with Rh-PNP complex is similar to that over Rh-PNP/ND. Unligated $[\mathrm{Rh}(\mathrm{COD}) \mathrm{Cl}]_{2} / \mathrm{ND}$ prepared by directly immobilizing $[\mathrm{Rh}(\mathrm{COD}) \mathrm{Cl}]_{2}$ on $\mathrm{ND}$, gave much lower activity $\left(17 \%\right.$ conversion, and $\left.\mathrm{TOF}=27 \mathrm{~h}^{-1}\right)$ with inferior regioselectivity $(b / l=2.0)$. A conventional heterogeneous catalyst, $\mathrm{ND}$ supported $\mathrm{Rh}$ nanoparticles (denoted as Rh NPs/ND), only presented $11 \%$ conversion $\left(\mathrm{TOF}=15 \mathrm{~h}^{-1}\right)$ with a $b / l$ ratio of 1.9:1.

Isolated Rh sites with different coordination environment. According to several decades of intensive studies of infrared spectra of chemisorbed $\mathrm{CO}$ on $\mathrm{Rh}$, in situ CO-diffuse reflectance infrared Fourier transform spectroscopy (CO-DRIFTs) has been proved to be a powerful technology to distinguish the isolated $\mathrm{Rh}$ atoms from other types of $\mathrm{Rh}$ species ${ }^{33,44-48}$. The most notable characteristic of isolated $\mathrm{Rh}$ species is a well-resolved doublet of chemisorbed $\mathrm{CO}$, which was assigned to symmetric and antisymmetric coupling between gem-dicarbonyl $\mathrm{Rh}(\mathrm{CO})_{2}$ on isolated $\mathrm{Rh}$ species or Rh complex compounds. For $\mathrm{Rh}_{1} / \mathrm{PNP}-\mathrm{ND}$, a well-resolved doublet with components at 2080 and $2007 \mathrm{~cm}^{-1}$ was exclusively observed in Fig. 5, indicating the atomically dispersed $\mathrm{Rh}$. In addition, as purging time increased, the position of the doublet for keeps invariant with the decrease in peak intensity (Supplementary Fig. 5). This phenomenon is a distinctive feature of gem-dicarbonyl $\mathrm{Rh}(\mathrm{CO})_{2}$ on isolated $\mathrm{Rh}$ atoms. Similar doublet with components at high wavenumbers $\left(2085\right.$ and $\left.2014 \mathrm{~cm}^{-1}\right)$ was also observed on $[\mathrm{Rh}(\mathrm{COD}) \mathrm{Cl}]_{2} / \mathrm{ND}$ (Fig. 5 and Supplementary Fig. 7), suggesting the nature of isolated $\mathrm{Rh}$ species. RhPNP/ND presented a dominant doublet at 2084 and $2013 \mathrm{~cm}^{-1}$ and a very weak prak at $2021 \mathrm{~cm}^{-1}$ (Fig. 5 and Supplementary Fig. 6), which clearly revealed that the majority of $\mathrm{Rh}$ species existed in the form of isolated sites. For the Rh NPs/ND, a 
Table 1 Screening of various Rh catalysts in the hydroformylation of styrene.

\begin{tabular}{|c|c|c|c|c|c|c|c|}
\hline Entry & Catalyst & Rh loading (wt\%) & $t(h)$ & Conv. (\%) & Sel. ${ }^{a}{ }_{C H O}(\%)$ & $b /\left.\right|^{b}$ & TOF $\left(h^{-1}\right)$ \\
\hline 1 & $\mathrm{Rh}_{1} / \mathrm{PNP}-\mathrm{ND}$ & 0.50 & 10 & 77 & $>99$ & $12.4: 1$ & 95 \\
\hline $2^{c}$ & {$[\mathrm{Rh}(\mathrm{COD}) \mathrm{Cl}]_{2}-\mathrm{PNP}$} & / & 10 & 85 & 99 & $4.9: 1$ & 105 \\
\hline 3 & Rh-PNP/ND & 0.28 & 10 & 44 & 98 & $4.7: 1$ & 96 \\
\hline 4 & {$[\mathrm{Rh}(\mathrm{COD}) \mathrm{Cl}]_{2} / \mathrm{ND}$} & 0.37 & 10 & 17 & 94 & $2.0: 1$ & 27 \\
\hline 5 & Rh NPs/ND & 0.40 & 10 & 11 & 91 & $1.9: 1$ & 15 \\
\hline 6 & $\mathrm{Rh}_{1} / \mathrm{PNP}-\mathrm{ND}$ & 0.50 & 16 & $>99$ & $>99$ & $13.1: 1$ & 77 \\
\hline $7 d$ & $\mathrm{Rh}_{1} / \mathrm{PNP}-\mathrm{ND}$ & 0.50 & 30 & $>99$ & $>99$ & $11.9: 1$ & 37 \\
\hline
\end{tabular}

Reaction conditions: $3 \mathrm{mmol}$ styrene, $50 \mathrm{mg}$ catalyst, $15 \mathrm{~mL}$ toluene, $15 \mathrm{~mL} \mathrm{H} \mathrm{H}_{2} \mathrm{O} 3.0 \mathrm{MPa}$ syngas $\left(\mathrm{CO} / \mathrm{H}_{2}=1\right), 50^{\circ} \mathrm{C}$, The Rh loading was determined by ICP-AES.

aThe selectivity for aldehydes products.

bThe molar ratio of branched aldehydes/linear aldehydes.

The molar ratio of $\mathrm{Rh} / \mathrm{PNP}$ is $1: 1,0.25 \mathrm{mg}$ Rh was used.

d $200 \mathrm{mg}$ catalyst, $3 \mathrm{~mL} \mathrm{H} \mathrm{H}_{2} \mathrm{O}, 12 \mathrm{mmol}$ styrene $(1.42 \mathrm{~mL}$ )



Fig. $\mathbf{5}$ In situ CO-DRIFTs spectra of Rh catalysts. Prior to characterization, all the samples were pretreated under helium at $200^{\circ} \mathrm{C}$ for $1 \mathrm{~h}$, and then further treated in $1 \% \mathrm{CO} / \mathrm{He}$ for $1 \mathrm{~h}$. A series of infrared spectra curves for $\mathrm{Rh}_{1} / \mathrm{PNP}-\mathrm{ND}$, Rh-PNP/ND, $\left[\mathrm{Rh}(\mathrm{COD}) \mathrm{Cl}_{2} / \mathrm{ND}\right.$, and Rh NPs/ND were measured over time (see Supplementary Information for detail).

doublet at relatively low wavenumbers $\left(2075\right.$ and $\left.2011 \mathrm{~cm}^{-1}\right)$, with a overlapped peak centered at $2036 \mathrm{~cm}^{-1}$ (Fig. 5 and Supplementary Fig. 8). The former was due to the reduced Rh single atoms, while the later was attributed to the linear CO on metallic $\mathrm{Rh}$ cluster. This implied that Rh species on Rh NPs/ND existed in a mixture of isolated Rh atoms and Rh clusters. Such observation is in consistent with the XPS results, which revealed that Rh species on Rh NPs was in metallic states (Supplementary Fig. 4).

The detailed information about CO-DRIFTs was summarized in Supplementary Table 4. According to the wavenumbers of the doublet for dicarbonyl $\mathrm{Rh}(\mathrm{CO})_{2}$ in Fig. 5, the electronic density of the isolated $\mathrm{Rh}$ species was in an order of $\mathrm{Rh}_{1} / \mathrm{PNP}-\mathrm{ND}>\mathrm{Rh}$ $\mathrm{PNP} / \mathrm{ND}>[\mathrm{Rh}(\mathrm{COD}) \mathrm{Cl}]_{2} / \mathrm{ND}$. This trend was further confirmed by XPS spectra of Rh $3 d$ in Supplementary Figs. 1-4. The peaks of $\mathrm{Rh} 3 d_{5 / 2}$ in Rh-PNP/ND, and [Rh(COD)Cl] $]_{2} / \mathrm{ND}$ were centered at 309.9 , and $310.0 \mathrm{eV}$, respectively (Supplementary Figs. 2 and 3), which were higher than those in $\mathrm{Rh}_{1} / \mathrm{PNP}-\mathrm{ND}$ (309.8 and $308.7 \mathrm{eV}$ ). The higher electron density of $\mathrm{Rh}$ single atoms on $\mathrm{Rh}_{1} /$ PNP-ND was attributed to the obvious electron transfer from PNP ligand to Rh single atom through coordination. From the ratio of integrated absorbance of asymmetric and symmetric stretches, the angle $(2 a)$ between carbonyl groups on the isolated $\mathrm{Rh}$ atoms was calculated by Paul's formula ${ }^{45}$. The angle (2a) between carbonyl groups on $\mathrm{Rh}_{1} / \mathrm{PNP}-\mathrm{ND}$ is calculated to be $104^{\circ}$, while those on Rh-PNP/ND, $[\mathrm{Rh}(\mathrm{COD}) \mathrm{Cl}]_{2} / \mathrm{ND}, \mathrm{Rh}$ NPs/ $\mathrm{ND}$, and $\mathrm{Rh}_{1} / \mathrm{ZnO}$ are 120 , and 105,89 , and $90^{\circ}$, respectively, suggesting the geometry of $\mathrm{Rh}(\mathrm{CO})_{2}$ are closely associated with the coordination environment of the different isolated $\mathrm{Rh}$ atoms.

Origin of regioselectivity. According to the above results, we concluded that the geometry, the electronic states, and the coordination environment of $\mathrm{Rh}$ species had crucial effect on the regioselectivity. $\mathrm{Rh}$ single atoms on $\mathrm{Rh}_{1} / \mathrm{PNP}-\mathrm{ND}$ exhibited relatively high electron density, and big angle between gemdicarbonyl $\mathrm{Rh}(\mathrm{CO})_{2}$, which was closely associated with the excellent branched-regioselectivity. The isolated $\mathrm{Rh}$ species on $\mathrm{Rh}-\mathrm{PNP} / \mathrm{ND}$ presented bigger angle $\left(2 \mathrm{a}=120^{\circ}\right)$ between gemdicarbonyl $\mathrm{Rh}(\mathrm{CO})_{2}$, but were more positively charged. As a consequence, $\mathrm{Rh}-\mathrm{PNP} / \mathrm{ND}$ gave a moderate regioselectivity $(b / l$ ratio $=4.7: 1)$. The more positively charged isolated $\mathrm{Rh}$ species on $[\mathrm{Rh}(\mathrm{COD}) \mathrm{Cl}]_{2} / \mathrm{ND}$ exhibited even lower regioselectivity (b/l ratio of 2.0:1), albeit with a similar angle $\left(2 \mathrm{a}=105^{\circ}\right)$ compared to $\mathrm{Rh}_{1} /$ PNP-ND. Different from the positively charged Rh single atoms on the above three catalysts, non-regioselective $\mathrm{Rh}_{1} / \mathrm{ZnO}$-nanowires possessing slightly negative charged $\mathrm{Rh}$ single atoms, gave a small angle $\left(2 \mathrm{a}=94^{\circ}\right)$. This may be responsible for the loss in regioselectivity control. Metallic Rh NPs/ND showed quite inferior regioselectiviy and activity $(b / l$ ratio $=1.9: 1$ with TOF of $15 \mathrm{~h}^{-1}$ ) due to the formation of $\mathrm{Rh}$ clusters during hydrogen reduction.

DFT calculation. In order to figure out the role of the coordination chemistry of $\mathrm{Rh}$ single atoms in $\mathrm{Rh}_{1} / \mathrm{PNP}-\mathrm{ND}$ in determining the regioselectivity of the hydroformylation reaction, density functional theory (DFT) calculations were performed. The energy files for the reaction pathways of branched and linear products was illustrated in Fig. 6 . The $\mathrm{Rh}_{1} / \mathrm{PNP}-\mathrm{ND}$ model was established by coordinating one $\mathrm{Rh}$ atom with two $\mathrm{P}$ atoms on a graphene-like monolayer (Supplementary Fig. 12). A 4-coordinate square-planar structure with two $\mathrm{P}$ atoms 1 was confirmed after optimization. The average Rh-P length in $\mathbf{1}$ is $2.31 \AA$, which is in line with EXAFS results $(2.38 \AA)$. The barrier of styrene coordination on 5-coordinate Rh single atom 




Fig. 6 Free energy profiles of the branched, and linear reaction pathways for styrene hydroformylation. All of the calculations were performed with Gaussian09 package (see Supplementary Information for detail). Geometry optimization of all the minima was carried out at the B3LYP level with the 6-31 G* basis set for $\mathrm{C}, \mathrm{H}, \mathrm{N}, \mathrm{O}, \mathrm{P}$ and Lanl2DZ for Rh. Default convergence criteria were used.

TS1-2 was $0.19 \mathrm{eV}$. For the linear, and branched reaction pathways, the coordination of styrene were in fact identical. The hydride insertion was crucial in determining the regioselectivity. The reaction barrier of the hydride insertion state for the branched pathway was $0.69 \mathrm{eV}$, lower than that for the linear pathway $(0.74 \mathrm{eV})$. The reaction energy for the linear-, and branched- alkyl complex was 0.09 and $-0.01 \mathrm{eV}$, respectively. The relative rates for the branched and linear reaction pathways is calculated to be 5.75 and 1.00 , which resulted in a predicted ratio of branched to linear selectivity of 85:15. This result matched toughly with the experimental data (in the range of 90:10-95:5), supporting the finding that hydride transfer from $\mathrm{Rh}$ single atom to adsorbed styrene played a determining role in the regioselectivity. For the formation of alkyl complex from $2, \Delta \mathrm{G}$ for the branched alkyl complex 4 is $-3.36 \mathrm{kcal} \mathrm{mol}^{-1}$, while $\Delta \mathrm{G}$ for the linear one 3 is $-1.21 \mathrm{kcal} \mathrm{mol}^{-1}$, indicating the branched reaction pathway was also thermodynamically favorable. Therefore, the coordination environment of $\mathrm{Rh}$ single atom in $\mathrm{Rh}_{1} / \mathrm{PNP}-\mathrm{ND}$ favored the branched reaction pathway thermodynamically and kinetically, which was responsible for the excellent regioselectivity towards the branched aldehydes.

Stability test. The time-conversion/regioselectivity curves in Fig. 7a showed full conversion of styrene was achieved with high regioselectivity $(b / l>12)$ within $16 \mathrm{~h}$. Temperature effect on the activity and regioselectivity was illustrated in Fig. 7b. The regioselectivity decreased with the increase in temperatures. Then the stability of $\mathrm{Rh}_{1} / \mathrm{PNP}-\mathrm{ND}$ was investigated in biphasic hydroformylation of styrene. Under a controlled conversion of $77 \%, \mathrm{Rh}_{1} / \mathrm{PNP}-\mathrm{ND}$ could be recycled at least 6 times without loss in activity and regioselectivity, demonstrating the good stability (Fig. 7c). Furthermore, to rule out the possibility of Rh leaching, hot filtration experiment was conducted at $8 \mathrm{~h}$. No further conversion was observed after removing $\mathrm{Rh}_{1} / \mathrm{PNP}-\mathrm{ND}$, suggesting that the reaction exclusively proceeded heterogeneously. The XPS spectrum of $\mathrm{Rh} 3 d$ show the two peaks at 309.9 and $308.7 \mathrm{eV}$ (Fig. 7d), which was in consistent with those on the fresh catalyst, indicating the electronic states of $\mathrm{Rh}$ single atoms were unchanged after recycling. The AC-HAADF-STEM images of the used $\mathrm{Rh}_{1} / \mathrm{PNP}-\mathrm{ND}$ reveal that $\mathrm{Rh}$ species still existed in the form of single atoms after catalytic runs, and no aggregation or Rh cluster was observed, indicating the robust nature of $\mathrm{P}$-coordinated $\mathrm{Rh}$ single atoms (Supplementary Fig. 9). To our delight, $\mathrm{Rh}_{1} / \mathrm{PNP}$ ND was exclusively dispersed in aqueous phase, after reaction the

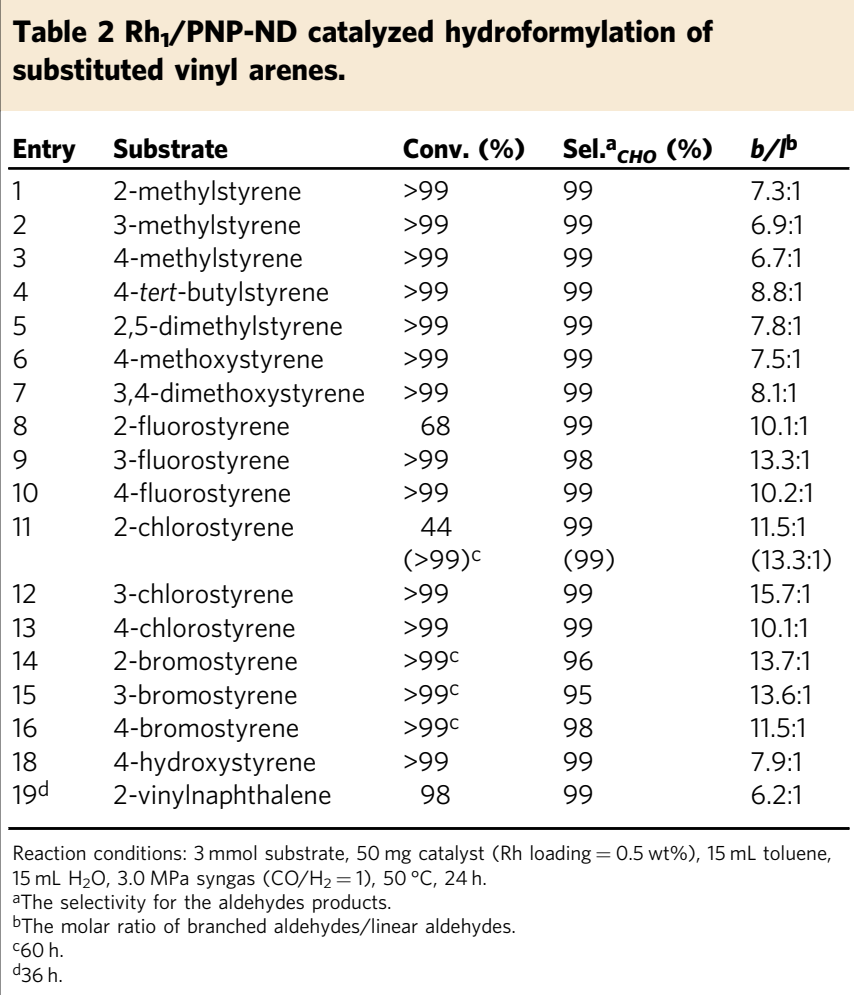

catalyst was spontaneously separated from the reaction mixtures (Fig. 2b). Benefitting from the hydrophilic nature of $\mathrm{Rh}_{1} / \mathrm{PNP}$ $\mathrm{ND}$, we conducted the reaction under an organic solvent-free conditions. As shown in Table 1 , the present $\mathrm{Rh}_{1} / \mathrm{PNP}-\mathrm{ND}$ still displayed high regioslectivity $(b / l$ ratio $=11.9: 1)$ with a completed conversion with a prolonged reaction time of $30 \mathrm{~h}$ in water.

Substrate scope. The substrate scope was explored to evaluate the generality of this catalytic system. Table 2 illustrated that vinyl arenes could be transformed to the corresponding aldehydes with excellent chemoselectivities in all cases. Most substituted vinyl arenes exhibited $>99 \%$ conversion with excellent regioselectivities ( $>90 \%$ ), indicating the high efficiency of $\mathrm{Rh}_{1} / \mathrm{PNP}-\mathrm{ND}$. However, sterically crowded 2-fluorostyrene and 2-chlorostyrene gave 68 and $44 \%$ conversion within $24 \mathrm{~h}$, respectively, indicating the strong steric hindrance effect of ortho- substituents on activity. Further extending reaction time to $60 \mathrm{~h}$ afforded full conversion of 2-chlorostyrene with high regioselectivity $(b / l=13.1)$. The electronic effect of the substitutes on regioselectivity was also proven. For instance, para-methyl- and para-methoxy-substituted vinylarenes afforded relatively low regioselectivities towards the corresponding branched aldehydes, respectively. 2Vinylnaphthalene was considered as hardly reactive in toluene/ water biphase system because of the large steric hindrance and the low water solubility ${ }^{49-52}$. To our delight, 2-vinylnaphthalene was also quite reactive in the present system, giving $98 \%$ conversion, high chemoselectivity $(>99 \%)$ and a $b / l$ ratio of $6.2: 1$ with prolonged time $(36 \mathrm{~h})$.

For vinyl arenes with insufficient water solubility, the hydroformylation slowly occurred at the water/organic interface and severely limited by mass transport. In the present work, PNPND worked as an effective mass transfer promoter for its hydrophobic core and hydrophilic surface. Due to this reason, insoluble vinyl arenes were readily diffused onto the surface of $\mathrm{Rh}_{1} / \mathrm{PNP}-\mathrm{ND}$, and then migrated to the highly dispersible single 

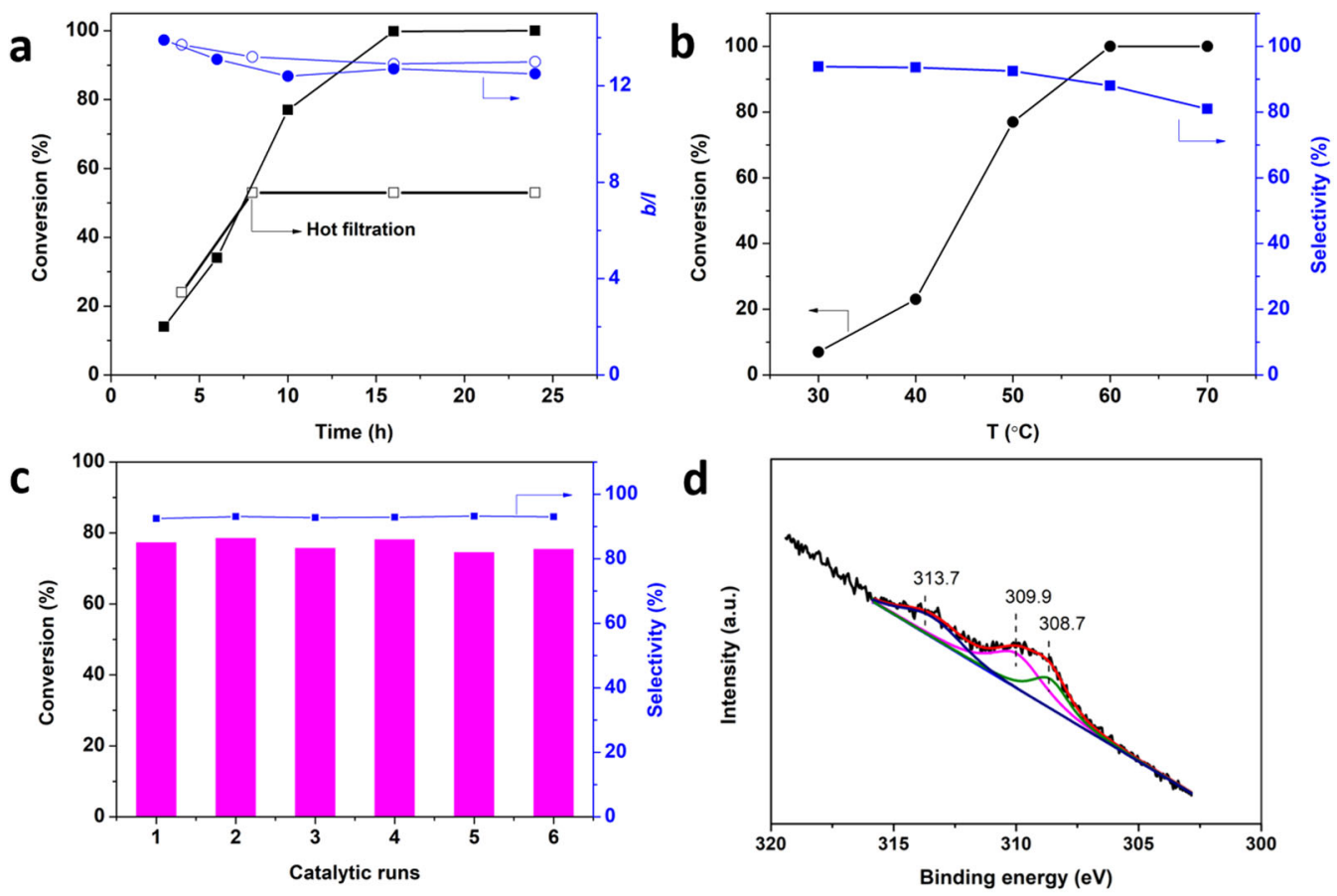

Fig. 7 Catalytic performance and characterization of $\mathbf{R h}_{\mathbf{1}} / \mathbf{P N P}$-ND in stability test. All the experiments were conducted under the reaction conditions of $3 \mathrm{mmol}$ styrene, $50 \mathrm{mg}$ catalyst, $15 \mathrm{~mL}$ toluene, $15 \mathrm{~mL} \mathrm{H} \mathrm{H}_{2} \mathrm{O}, 3.0 \mathrm{MPa}$ syngas $\left(\mathrm{CO} / \mathrm{H}_{2}=1\right)$. a Time-conversion \& regioselectivity curve. Reaction conditions: $50^{\circ} \mathrm{C}$. b Effect of reaction temperature on the catalytic performance. Reaction conditions: $10 \mathrm{~h}$. c Stability test. Reaction conditions: $50{ }^{\circ} \mathrm{C}, 10 \mathrm{~h}$. Recovered catalyst was washed with ultra-pure water, centrifuged, and submitted to the next run. d XPS survey spectra of Rh $3 d$ for the used Rh 1 PNP-ND after six runs.

$\mathrm{Rh}$ atom sites. Thus, the reaction rate was greatly accelerated in consequence.

Additionally, terminal aliphatic alkenes $\left(\mathrm{C}_{6}-\mathrm{C}_{10}\right)$ were also explored to evaluate the regioselectivity of $\mathrm{Rh}_{1} / \mathrm{PNP}-\mathrm{ND}$. It is well known that hydroformylation of terminal aliphatic alkenes usually gave linear aldehydes in high regioselectivity $(b / l<0.1)$, and the branched-selective hydroformylation of terminal aliphatic alkenes was highly challenging. The most recent work was reported by Nozaki and co-workers ${ }^{53}$. With the specifically designed nitrogencentered triphosphine ligand, the corresponding $\mathrm{Rh}$ complex catalysts $(1.0 \mathrm{~mol} \% \mathrm{Rh})$ gave a $b / l$ ratio of 1.1 with completed conversion in the hydroformylation of 1 -hexene at $100^{\circ} \mathrm{C}, 3.5 \mathrm{~h}$, and $2.0 \mathrm{MPa}$. In the present work, $\mathrm{Rh}_{1} / \mathrm{PNP}-\mathrm{ND}$ exhibited the $b / l$ ratios in the range of $0.58-0.78$ (Supplementary Table 5), indicating the branched-selective nature of $\mathrm{Rh}_{1} / \mathrm{PNP}-\mathrm{ND}$.

Applications in pharmaceutical synthesis. The application of heterogeneous single-atom catalysis in pharmaceutical synthesis was rarely reported in the previous literatures ${ }^{40}$. Herein, we conducted two synthetic transformations for preparing two pharmaceutical molecules Ibuprofen and Fendiline, in order to demonstrate the practicability of the present $\mathrm{Rh}_{1} / \mathrm{PNP}-\mathrm{ND}$-catalyzed hydroformylation. As depicted in Fig. 8a, a gram-scale of hydroformylation of 4-isobutylstyrene was efficiently conducted, giving the target branched aldehyde of $92 \%$ yield. Then the formed intermediate aldehyde was subjected to mild aqueous oxidation $\left(\mathrm{NaClO}_{2}, \mathrm{KHPO}_{2}\right.$, 2-methyl-2-butene, $t$ - $\mathrm{BuOH}, \mathrm{H}_{2} \mathrm{O}, 0$ ${ }^{\circ} \mathrm{C}$ to r.t., $1 \mathrm{~h}$ ) in a highly selective manner ${ }^{54}$. Finally, Ibuprofen was obtained in an overall isolated yield of $85 \%$. Moreover, a convenient synthetic route to Fendiline was furnished with onepot hydroformylation/amination reaction (Fig. 8b). Remarkably, sterically hindered 1,1-diphenylethylene could be converted into the corresponding linear aldehyde in $91 \%$ yield, followed by reductive amination with 1-phenylethylamine in the presence of
4.0 $\mathrm{MPa} \mathrm{H}_{2}$. With this methodology the overall yield of Fendiline was as high as $87 \%$, which was comparable with that using homogeneous rhodium-carbene complexes ${ }^{55}$.

\section{Discussion}

In summary, we have developed a P-coordinated Rh single-atom catalyst $\left(\mathrm{Rh}_{1} / \mathrm{PNP}-\mathrm{ND}\right)$ by using dispersable nanodiamond as support. Systematic investigation revealed that each isolated $\mathrm{Rh}$ atom was firmly anchored on nanodiamond through the bidentate chelation with two $\mathrm{P}$ atoms. The electron transfer from the grafted phosphorus ligand to Rh single atom was confirmed by in situ CO-DRIFTs and XPS. This unique structure was analogous to homogeneous Rh-P complex catalysts, and highly related to the remarkable catalytic performance of $\mathrm{Rh}_{1} / \mathrm{PNP}-\mathrm{ND}$ in hydroformylation of a series of vinyl arenes. Consequently, diverse $\alpha$-arylpropionaldehydes, including two important drug intermediates, were produced with excellent yields in a green and efficient way. Our work opened an important prospect of singleatom catalyst in pharmaceutical synthesis.

\section{Methods}

Synthesis of PNP pincer ligand Bis[2-(diphenylphosphino)ethy]amine. Under nitrogen atmosphere, diphenylphosphine $(14.0 \mathrm{~mL}, 80 \mathrm{mmol})$ was added to a solution of tert-butoxide (14 g, $125 \mathrm{mmol})$ in anhydrous THF (300 ml) at ambient temperature. And then bis(2-chloroethyl)amine hydrochloride $(7.15 \mathrm{~g}, 40 \mathrm{mmol})$ was added to the solution. The mixture was stirred for $18 \mathrm{~h}$ under reflux. After reaction, the reaction mixture was poured into $400 \mathrm{~mL}$ hexane, the organic phase was washed with $10 \%$ aqueous $\mathrm{NaOH}$, saturated aqueous $\mathrm{NaCl}$ solution, and $2 \mathrm{~N}$ aqueous $\mathrm{HCl}$ solution to provide a dense white precipitate of $\mathbf{1} \cdot \mathrm{HCl}$. Recrystallization from boiling acetonitrile gave a $90 \%$ yield $(17.2 \mathrm{~g})$ of $\mathbf{1} \cdot \mathrm{HCl}$ of white needles. ${ }^{1} \mathrm{H}$ NMR $\left(400 \mathrm{MHz}, \mathrm{CDCl}_{3}\right): \delta 2.3-3.3(\mathrm{~m}, 8 \mathrm{H}), 7.0-7.6(\mathrm{~m}, 20 \mathrm{H}), 9.9(\mathrm{~s}, 2 \mathrm{H})$.

Synthesis of $\mathbf{R h}_{\mathbf{1}} / \mathbf{P N P}-\mathbf{N D}$. Nanodiamond (Nanjing XFNANO Materials Tech Co.Ltd), diphosphino-amine, CDMT (2-Chloro-4,6-dimethoxy-1,3,5-triazine), NMM (N-methyl morpholine), DCC (N,N'-dicyclohexylcarbodiimide), DMAP(4Dimethylaminopyridine), TEA (triethylamine) were obtained from commercial sources and used without further purification. In step 1, nanodiamond support 
a) Gram scale synthesis of Ibuprofen via $\mathrm{Rh}_{1} / \mathrm{PNP}-\mathrm{ND}$ catalyzed hydroformylation<smiles>C=Cc1ccc(CC(C)C)cc1</smiles>

b) One-pot synthesis of Fendiline via tandam hydroformylation/amination catalyzed by $\mathrm{Rh}_{\mathbf{1}} / \mathrm{PNP}$-ND<smiles>C=C(c1ccccc1)c1ccccc1</smiles>

$3 \mathrm{mmol}$<smiles>CCC(CC)c1ccccc1</smiles>

$93 \%$ yield



$94 \%$ yield<smiles>NC(NCCC(c1ccccc1)c1ccccc1)c1ccccc1</smiles>

Fendiline

Fig. 8 The synthetic routes for Ibuprofen and Fendiline via Rh1/PNP-ND catalyzed hydorformylation. a For the hydroformylation step, $200 \mathrm{mg}$ Rh $/$ /PNP$\mathrm{ND}$ (Rh loading $=0.5 \mathrm{wt} \%$ ), $30 \mathrm{~mL}$ toluene, $30 \mathrm{~mL} \mathrm{H} 2 \mathrm{O}, 40^{\circ} \mathrm{C}, 24 \mathrm{~h}, 3.0 \mathrm{MPa} \mathrm{CO} / \mathrm{H}_{2}$, isolated yield; for the oxidation step, $20 \mathrm{~mL} t-\mathrm{BuOH}, 15 \mathrm{~mL}$ aq. $\mathrm{NaH}_{2} \mathrm{PO}_{4}$ solution $(1.70 \mathrm{M})$, and $15 \mathrm{~mL}$ aq. $\mathrm{NaClO}_{2}$ solution $(1.0 \mathrm{M}), 1 \mathrm{~h}$, isolated yield. $\mathbf{b}$ for hydroformylation step, $300 \mathrm{mg} R \mathrm{R}_{1} / \mathrm{PNP}-\mathrm{ND}(\mathrm{Rh}$ loading $=0.5$ wt\%), $15 \mathrm{~mL}$ toluene, $15 \mathrm{~mL} \mathrm{H} \mathrm{O}_{1}, 110^{\circ} \mathrm{C}, 24 \mathrm{~h}, 3.0 \mathrm{MPa} \mathrm{CO} / \mathrm{H}_{2}\left(\mathrm{CO} / \mathrm{H}_{2}=2\right), \mathrm{GC}$ yield; for reductive amination step, $300 \mathrm{mg} \mathrm{Rh} / \mathrm{PNP}-\mathrm{ND}(\mathrm{Rh}$ loading $=$ 0.5 wt\%), 1.1 eq. 1-phenylethylamine, $30 \mathrm{~mL}$ ethanol, $120^{\circ} \mathrm{C}, 24 \mathrm{~h}, 4.0 \mathrm{MPa} \mathrm{H}_{2}$, isolated yield.

$(50 \mathrm{mg})$ was dispersed into solvent $(12 \mathrm{~mL})$ by ultrasonic treatment for $5 \mathrm{~min}$. And then diphosphino-amine $(88 \mathrm{mg}, 0.185 \mathrm{mmol})$, CDMT $(32 \mathrm{mg}, 0.185 \mathrm{mmol})$, and NMM ( $65 \mathrm{mg}, 0.65 \mathrm{mmol}$ ) were added into the mixture. The slurry was stirred at $90{ }^{\circ} \mathrm{C}$ for $48 \mathrm{~h}$ under nitrogen atmosphere. After centrifugation, the residual solid washed for three times with DMF and THF, respectively. The sample was dried at $50^{\circ} \mathrm{C}$ under vacuum overnight, denoted as PNP-ND.

In step $2,50 \mathrm{mg}$ PNP-ND and $46 \mathrm{mg}\left[\mathrm{Rh}(\mathrm{COD}) \mathrm{Cl}_{2}\right.$ were added in $10 \mathrm{~mL}$ solvent, and then stirred at $30^{\circ} \mathrm{C}$ for $12 \mathrm{~h}$ under $\mathrm{N}_{2}$. After that, the mixture was centrifugated, and washed with THF for three times. The solid was dried under vacuum at $50^{\circ} \mathrm{C}$ overnight, and then pretreated in hydrogen at $120^{\circ} \mathrm{C}$ for $2 \mathrm{~h}$. The obtained sample was denoted as $\mathrm{Rh}_{1} / \mathrm{PNP}-\mathrm{ND}$.

Catalysts characterization. Solid-state ${ }^{31} \mathrm{P}$ NMR spectra were performed on a Bruker 400WB AVANCE III NMR spectrometer with a magnetic field strength of $9.4 \mathrm{~T}$. The metal loading was analyzed by inductively coupled plasma atomic emissionspectroscopy (IRIS Intrepid II XSP, Thermo Electron). STEM characterization was conducted with a JEOL JEM-2100F instrument at $200 \mathrm{kV}$. The ACHAADF-STEM images was collected on a JEOL JEM-ARM200F operated at $300 \mathrm{kV}$, with a guaranteed resolution of $80 \mathrm{pm}$. The X-ray absorption spectra including X-ray absorption near-edge structure (XANES) and extended X-ray absorption fine structure (EXAFS) at the K-edge of Rh of the samples were collected at the BL 14W1 of Shanghai Synchrotron Radiation Facility (SSRF), China. The Ru foil was employed to calibrate the energy. The spectra were collected under transmission mode at room temperature. The Athena software package was used to analyze the data. In situ CO-diffuse reflectance infrared Fourier transform spectroscopy (DRIFTS) was measured in the range $4000-450 \mathrm{~cm}^{-1}$ on a Nicolet 6700 FTIR spectrophotometer (Thermo Fisher). The Rh content in Rh samples and in reaction mixture was determined using an Agilent 730 ICP-OES. X-ray photoelectron spectra (XPS) were performed on an ESCALAB 250 X-ray photoelectron spectrometer equipped with a monochromated $\mathrm{Al} \mathrm{Ka}$ anode. ${ }^{1} \mathrm{H}$ NMR spectra were recorded at room temperature on $400 \mathrm{MHz}$ Bruker DRX-400 NMR spectrometers.

Hydroformylaion reaction. The hydroformylation reaction was conducted in a $50 \mathrm{~mL}$ high-pressure autoclave. In a typical run, $50 \mathrm{mg}$ catalyst, $3 \mathrm{mmol}$ substrate, $15 \mathrm{~mL}$ toluene and $15 \mathrm{~mL}$ ultra-pure water were loaded. And then the reactor was purged with syngas $\left(\mathrm{CO} / \mathrm{H}_{2}=1\right)$, and charged with high-pressure syngas. The reactor was heated to the desired temperature with stirring. After reaction the reaction products were determined by GC/MSD instrument (Agilent 7820A/5977B, equipped with a HP-5 column), and quantified by GC (GC-FID, Agilent 6890A equipped with a DB-624 column).

Experimental procedures for catalyst preparation, characterization, and activity test are provided in Supplementary Information in detail.

\section{Data availability}

The data supporting the findings of this study are available from the article and the supplementary information, or from the corresponding authors upon reasonable request.
Received: 14 February 2021; Accepted: 12 July 2021; Published online: 04 August 2021

\section{References}

1. Franke, R. et al. Applied hydroformylation. Chem. Rev. 112, 5675-5732 (2012).

2. Nurttila, S. et al. Supramolecular approaches to control activity and selectivity in hydroformylation catalysis. ACS Catal. 8, 3469-3488 (2018).

3. Kalck, P. et al. Tandem hydroaminomethylation reaction to synthesize amines from alkenes. Chem. Rev. 118, 3833-3861 (2018).

4. Liu, Q. et al. Using carbon dioxide as a building block in organic synthesis. Nat. Commun. 6, 5933-5948 (2015).

5. Wu, X. et al. Transition-metal-catalyzed carbonylation reactions of olefins and alkynes: a personal account. Acc. Chem. Res. 47, 1041-1053 (2014).

6. You, C. et al. Design and application of hybrid phosphorus ligands for enantioselective Rh-catalyzed anti-Markovnikov hydroformylation of unfunctionalized 1,1-disubstituted alkenes. J. Am. Chem. Soc. 140, 4977-4981 (2018).

7. Ren, X. et al. Rhodium-complex-catalyzed hydroformylation of olefins with $\mathrm{CO}_{2}$ and hydrosilane. Angew. Chem. Int. Ed. 56, 310-313 (2017).

8. You, X. et al. Rhodium-catalyzed desymmetrization by hydroformylation of cyclopentenes: synthesis of chiral carbocyclic nucleosides. Angew. Chem. Int. Ed. 55, 6511-6514 (2016).

9. You, C. et al. Enantioselective Rh-catalyzed anti-Markovnikov hydroformylation of 1,1-disubstituted allylic alcohols and amines: an efficient route to chiral lactones and lactams. ACS Catal. 9, 8529-8533 (2019).

10. Phanopoulos, A. et al. Branched-selective hydroformylation of nonactivated olefins using an N-triphos/Rh catalyst. ACS Catal. 8, 5799-5809 (2018).

11. Dingwall, P. et al. Understanding a hydroformylation catalyst that produces branched aldehydes from alkyl alkenes. J. Am. Chem. Soc. 139, 15921-15932 (2017).

12. Terhorst, M. et al. One-pot synthesis of aldoximes from alkenes via Rhcatalysed hydroformylation in an aqueous solvent system. Green. Chem. 22, 7974-7982 (2020)

13. Tudor, T. et al. Industrial low pressure hydroformylation: forty-five years of progress for the LP oxo SM process. Johns. Matthey Technol. Rev. 61, 246-256 (2017).

14. Kohlpaintner, C. et al. Aqueous biphasic catalysis: Ruhrchemie/Rh^onePoulenc oxo process. Appl. Catal. A: Gen. 221, 219-225 (2001).

15. Tudor, R. et al. Enhancement of industrial hydroformylation processes by the adoption of rhodium-based catalyst: Part II. Platin. Met. Rev. 51, 164-171 (2007).

16. Liao, J. et al. Transition-metal catalyzed asymmetric reactions under continuous flow from 2015 to early 2020. Green. Synth. Catal. 1, 121-133 (2020). 
17. Rupflin, L. et al. Platinum group metal phosphides as heterogeneous catalysts for the gas-phase hydroformylation of small olefins. ACS Catal. 7, 3584-3590 (2017).

18. Zhao, Y. et al. Hydroformylation of 1-octene in Pickering emulsion constructed by amphiphilic mesoporous silica nanoparticles. J. Catal. 334, 52-59 (2016).

19. Tan, $\mathrm{M}$. et al. $\mathrm{PPh}_{3}$ functionalized $\mathrm{Rh} / \mathrm{rGO}$ catalyst for heterogeneous hydroformylation: Bifunctional reduction of graphene oxide by organic ligand. Chem. Eng. J. 330, 863-869 (2017).

20. Wang, L. et al. Atomic-level insights in optimizing reaction paths for hydroformylation reaction over $\mathrm{Rh} / \mathrm{CoO}$ single-atom catalyst. Nat. Commun. 7, 14036-14044 (2016).

21. Sun, Q. et al. Highly efficient heterogeneous hydroformylation over Rhmetalated porous organic polymers: synergistic effect of high ligand concentration and flexible framework. J. Am. Chem. Soc. 137, 5204-5209 (2015).

22. $\mathrm{Vu}, \mathrm{T}$. et al. Selective hydroformylation of olefins over the rhodium supported large porous metal-organic framework MIL-101. Appl. Catal. A: Gen. 468, 410-417 (2013).

23. Cui, X. et al. Bridging homogeneous and heterogeneous catalysis by heterogeneous single-metal-site catalysts. Nat. Catal. 1, 385-397 (2018).

24. Yang, X. et al. Single-atom catalysts: a new frontier in heterogeneous catalysis. Acc. Chem. Res. 46, 1740-1748 (2013).

25. Huang, F. et al. Anchoring $\mathrm{Cu}_{1}$ species over nanodiamond-graphene for semihydrogenation of acetylene. Nat. Commun. 10, 4431 (2019).

26. Pei, G. X. et al. Ag alloyed Pd single-atom catalysts for efficient selective hydrogenation of acetylene to ethylene in excess ethylene. ACS Catal. 5, 3717-3725 (2015).

27. Qiao, B. et al. Single-atom catalysis of $\mathrm{CO}$ oxidation using $\mathrm{Pt}_{1} / \mathrm{FeO}_{\mathrm{x}}$. Nat. Chem. 3, 634-641 (2011).

28. Wei, $\mathrm{H}$. et al. $\mathrm{FeO}_{\mathrm{x}}$-supported platinum single-atom and pseudo-singleatom catalysts for chemoselective hydrogenation of functionalized nitroarenes. Nat. Commun. 5, 5634 (2014)

29. Chen, Z. et al. A heterogeneous single-atom palladium catalyst surpassing homogeneous systems for Suzuki coupling. Nat. Nanotechnol. 13, 702-707 (2018).

30. Lin, J. et al. Remarkable performance of $\mathrm{Ir}_{1} / \mathrm{FeO}_{\mathrm{x}}$ single-atom catalyst in water gas shift reaction. J. Am. Chem. Soc. 135, 15314-15317 (2013).

31. Liu, P. et al. Photochemical route for synthesizing atomically dispersed palladium catalysts. Science 352, 797-801 (2016).

32. Lin, L. et al. A highly CO-tolerant atomically dispersed Pt catalyst for chemoselective hydrogenation. Nat. Nanotechnol. 14, 354-361 (2019).

33. Lang, R. et al. Hydroformylation of olefins by a rhodium single-atom catalyst with activity comparable to $\mathrm{RhCl}\left(\mathrm{PPh}_{3}\right)_{3}$. Angew. Chem. Int. Ed. 55, 16054-16058 (2016).

34. Li, T. et al. Styrene hydroformylation with in situ hydrogen: regioselectivity control by coupling with the Low-Temperature Water-Gas Shift Reaction. Angew. Chem. Int. Ed. 59, 7430-7434 (2020)

35. Li, C. et al. Single atom dispersed Rh-biphephos\&PPh $@$ @porous organic copolymers: highly effi cient catalysts for continuous fixed-bed hydroformylation of propene. Green. Chem. 18, 2995-3005 (2016).

36. Wang, Y. et al. Highly efficient porous organic copolymer supported Rh catalysts for heterogeneous hydroformylation of butenes. Appl. Catal. A Gen. 551, 98-105 (2018).

37. $\mathrm{Li}, \mathrm{C}$. et al. Designing highly efficient $\mathrm{Rh} / \mathrm{CPOL}-\mathrm{bp} \& \mathrm{PPh}_{3}$ heterogenous catalysts for hydroformylation of internal and terminal olefins. Catal. Sci. Technol. 6, 2143-2149 (2016).

38. Wang, G. et al. Bifunctional heterogeneous Ru/POP catalyst embedded with alkali for the $\mathrm{N}$-formylation of amine and $\mathrm{CO}_{2}$. ACS sustain. Chem. Eng. 8 , 5576-5583 (2020).

39. Liu, B. et al. Promotion of inorganic phosphorus on Rh catalysts in styrene hydroformylation: geometric and electronic effects. ACS Catal. 11, 1787-1796 (2021).

40. Long, X. et al. Graphitic phosphorus coordinated single Fe atoms for hydrogenative transformations. Nat. Commun. 11, 4074 (2020).

41. Kuil, M. et al. Template-induced formation of heterobidentate ligands and their application in the asymmetric hydroformylation of styrene. Chem. Commun. 45, 4679-4681 (2006).

42. Zhang, H. et al. Effect of positive-charges in diphosphino-imidazolium salts on the structures of Ir-complexes and catalysis for hydroformylation. J. Mol. Catal. A: Chem. 411, 337-343 (2016).

43. Makhubela, B. et al. $\mathrm{Rh}(\mathrm{I})$ complexes supported on a biopolymer as recyclable and selective hydroformylation catalysts. Green. Chem. 14, 338-347 (2012).

44. Rupflin, L. et al. Platinum group metal phosphides as heterogeneous catalysts for the gas-phase hydroformylation of small olefins. ACS Catal. 7, 3584-3590 (2017).

45. Yates, J. et al. Infrared spectra of chemisorbed CO on Rh. J. Chem. Phys. 70, 1219-1224 (1979).
46. Cavanagh, $\mathrm{R}$. et al. Site distribution studies of $\mathrm{Rh}$ supported on $\mathrm{Al}_{2} \mathrm{O}_{3}-\mathrm{An}$ infrared study of chemisorbed CO. J. Chem. Phys. 74, 4150 (1981).

47. Goellner, F. et al. Structure and bonding of a site-isolated transition metal complex: rhodium dicarbonyl in highly dealuminated zeolite Y. J. Am. Chem. Soc. 122, 8056-8066 (2000).

48. Fielicke, A. et al. Size and charge effects on the binding of CO to small isolated rhodium clusters. J. Phys. Chem. B. 108, 14591-14598 (2004).

49. Pandey, S. et al. Iron catalyzed hydroformylation of alkenes under mild conditions: evidence of an Fe(II) catalyzed process. J. Am. Chem. Soc. 140, 4430-4439 (2018)

50. You, C. et al. Silicon-oriented regio- and enantioselective rhodium-catalyzed hydroformylation. Nat. Commun. 9, 2045-2054 (2018).

51. Guo, Y. et al. Synthesis of new triarylphosphine ligand and their application in styrene hydroformylation. Catal. Commun. 9, 1842-1845 (2008).

52. Allmendinger, S. et al. Easily accessible TADDOL-derived bisphosphonite ligands: synthesis and application in the asymmetric hydroformylation of vinylarenes. Adv. Synth. Catal. 357, 41-45 (2015).

53. Phanopoulos, A., \& Nozaki, K. Branched-selective hydroformylation of nonactivated olefins using an N-Triphos/Rh catalyst. ACS Catal. 8, 5799-5809 (2018).

54. Bal, B. et al. Oxidation of $\alpha, \beta$-unsaturated aldehydes. Tetrahedron 37, 2091-2096 (1981)

55. Ahmed, M. et al. Hydroaminomethylation with novel rhodium-carbene complexes: an efficient catalytic approach to pharmaceuticals. Chem. Eur. J. 13, 1594-1601 (2007).

\section{Acknowledgements}

We are grateful for financial support from the National Natural Science Foundation of China (21902032). G.L. acknowledges the funding support from Fudan University. The XAF experiments were performed in Shanghai Synchrotron Radiation Facility (SSRF).

\section{Author contributions}

P.G. and T.R. conducted catalyst synthesis and the catalytic tests. G.L. analyzed the data of catalyst characterizations. Y.L., H.Q. and Q.W. contributed to the X-ray absorption experiment and analyzed the data. G.L. and F.C. wrote the manuscript. G.L. and F.C. directed the project. All authors discussed the experimental results and commented on the manuscript.

\section{Competing interests}

The authors declare no competing interests.

\section{Additional information}

Supplementary information The online version contains supplementary material available at https://doi.org/10.1038/s41467-021-25061-0.

Correspondence and requests for materials should be addressed to G.L. or F.-E.C.

Peer review information Nature Communications thanks the anonymous reviewers for their contribution to the peer review of this work. Peer reviewer reports are available.

Reprints and permission information is available at http://www.nature.com/reprints

Publisher's note Springer Nature remains neutral with regard to jurisdictional claims in published maps and institutional affiliations.

\section{(i)}

Open Access This article is licensed under a Creative Commons Attribution 4.0 International License, which permits use, sharing, adaptation, distribution and reproduction in any medium or format, as long as you give appropriate credit to the original author(s) and the source, provide a link to the Creative Commons license, and indicate if changes were made. The images or other third party material in this article are included in the article's Creative Commons license, unless indicated otherwise in a credit line to the material. If material is not included in the article's Creative Commons license and your intended use is not permitted by statutory regulation or exceeds the permitted use, you will need to obtain permission directly from the copyright holder. To view a copy of this license, visit http://creativecommons.org/ licenses/by/4.0/.

(c) The Author(s) 2021 